\title{
160 Gbps Simulation of a Quantum Dot Semiconductor Optical Amplifier Based Optical Buffer
}

\author{
Maria Spyropoulou ${ }^{1}$,Konstantinos Yiannopoulos ${ }^{2}$, Stelios Sygletos ${ }^{1}$, \\ Kyriakos Vlachos ${ }^{2}$, Ioannis Tomkos ${ }^{1}$ \\ ${ }^{1}$ Athens Information Technology Centre, 19.5Km Markopoulou Av.Peania 19002, Greece \\ \{mspi,ssyg,itom\}@ait.edu.gr \\ ${ }^{2}$ Department of Computer Engineering and Informatics, and Research Academic Computer \\ Technology Institute, University of Patras, Rio 26500, Greece \\ \{giannopu, kvlachos\}@ceid.upatras.gr
}

\begin{abstract}
We demonstrate the applicability of quantum-dot semiconductor-opticalamplifier based wavelength converters to the implementation of an ultra-high speed optical packet switching buffer. The buffer architecture consists of cascaded programmable delay stages that fully utilize the available wavelengths and thus minimize the number of wavelength converters that are required to implement the buffer. Physical layer simulations demonstrate error-free operation of the buffer with 3 cascaded Time-Slot-Interchager (TSI) stages at $160 \mathrm{Gbps}$ in the $1.55 \mathrm{um}$ window.
\end{abstract}

Keywords: Optical buffers, Optical packet switching, Quantum Dot Semiconductor Optical Amplifiers, Wavelength Converters, Programmable Delays.

\section{Introduction}

Quantum dot semiconductor optical amplifiers (QDSOAs) are envisaged as the next generation optical signal processing devices in ultra high speed networks. This originates from their unique features such as high differential gain and ultra-fast gain recovery ( 100fs) [1], which make them superior to conventional bulk or quantum well (QW) devices. Moreover, their enhanced properties make QDSOAs ideal candidates for signal processing applications at line rates that reach $160 \mathrm{~Gb} / \mathrm{s}$.

In the current communication we demonstrate the applicability of QDSOAs in an optical buffer architecture that is suitable for ultra-high speed optical packet switching. Packet buffering is implemented in a multi-stage Time-Slot-Interchanger (TSI) that consists of QDSOA-based wavelength converters exploiting the cross-gain modulation (XGM) effect and feed-forward delay lines. Moreover, the TSI stages are designed to fully utilize the number of available wavelengths, which are defined in such a way to prevent spectral overlap in multi-hundred $\mathrm{Gb} / \mathrm{s}$ line rates and also to fit within the spectral width of homogeneous broadening of a single-dot group. The use of many wavelengths is critical when considering the hardware cost of the buffer architecture and the signal quality degradation due to successive wavelength conversions. The buffer architecture is accompanied by physical layer simulations that derive the achievable buffer size in terms of cascaded TSI stages and illustrate its efficient performance in terms of output Q-factor and extinction ratio. Simulations reveal that up to 3 TSI stages may be cascaded for error-free operation at $160 \mathrm{Gbps}$. 


\section{Buffer Architecture and Control}

\subsection{Buffer Architecture}

The proposed system architecture is presented in Fig. 1. It comprises cascaded programmable delay stages and each stage consists of two Tunable Wavelength Converters (TWCs) and two delay line banks. Each TWC provides $w$ separate wavelengths at its output, and each wavelength is routed to the respective delay line of the delay line bank by means of a wavelength de-multiplexer. Adjacent TWCs and stages are connected by wavelength multiplexers.

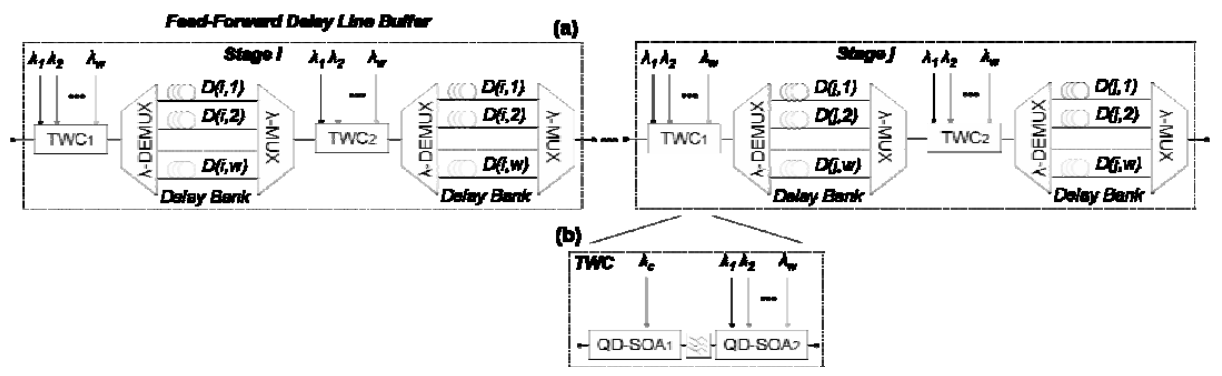

Fig. 1. (a) The structure of each delay stage. (b) The QDSOA tunable wavelength converter (TWC) setup. $\lambda$-MUX/DEMUX are the wavelength multiplexers and demultiplexers, respectively.

The delays that are introduced at each TSI stage are a design parameter of the proposed architecture and we will evaluate them from the timeslot transition graph (TTG) of the TSI architecture [2]. The TTG consists of nodes located at columns and rows; columns $i$ and $i+1$ correspond to the input and output, respectively, of TSI stage $i$, and rows correspond to timeslots, each being occupied by a single optical packet. An optical packet that has arrived at the input of stage $i$ and accesses one of the delay lines is placed in an output timeslot that appears later in time, and this action is represented as a straight line (time transition) connecting an input and an output node on the TTG.

Buffering a packet in the proposed design corresponds to a path on the TTG. The origin node represents the input-slot on which the packet has arrived and the destination node represents the output-slot where the packet leaves from. Taking into consideration that more that one packets arrive at the buffer inputs within a timeframe, it is evident that during each timeframe, an interconnection pattern which maps input to output nodes is formed on the TTG. The aim is to engineer the time transitions, or equivalently the delay times $D(i, j)$ at each stage, so that the interconnection pattern forms a $\log _{\mathrm{n}}$-Benes graph as a subgraph. The $\log _{n}$-Benes graph is derived from the $\log _{2}$-Benes graph after replacing the $2 \times 2$ with $n \times n$ switches. Furthermore, it is considered a well-known re-arrangeably nonblocking interconnection topology.

The purpose of constructing the $\log _{\mathrm{n}}$-Benes space-time graph is many-fold: the implementation requires a minimum number of serially connected stages that equals to

$$
s=2 \cdot m-1=2 \cdot\left\lceil\log _{n} N\right\rceil-1
$$


for buffering maximum $N$ number of packets. Eq. (1) shows that by implementing the $\log _{\mathrm{n}}$-Benes space-time graph, one can achieve a drastic reduction in the number of stages. This is of particular importance when considering the hardware cost of the implementation. Moreover, physical layer impairments aggravate the optical signal quality as the number of cascaded stages increases. An additional attribute of the Benes spacetime graph is that, it is re-arrangably non-blocking resulting in the capability of the proposed design to store packets without suffering internal collisions. Finally, finding collision-free paths within the Benes graph is a well studied problem [3].
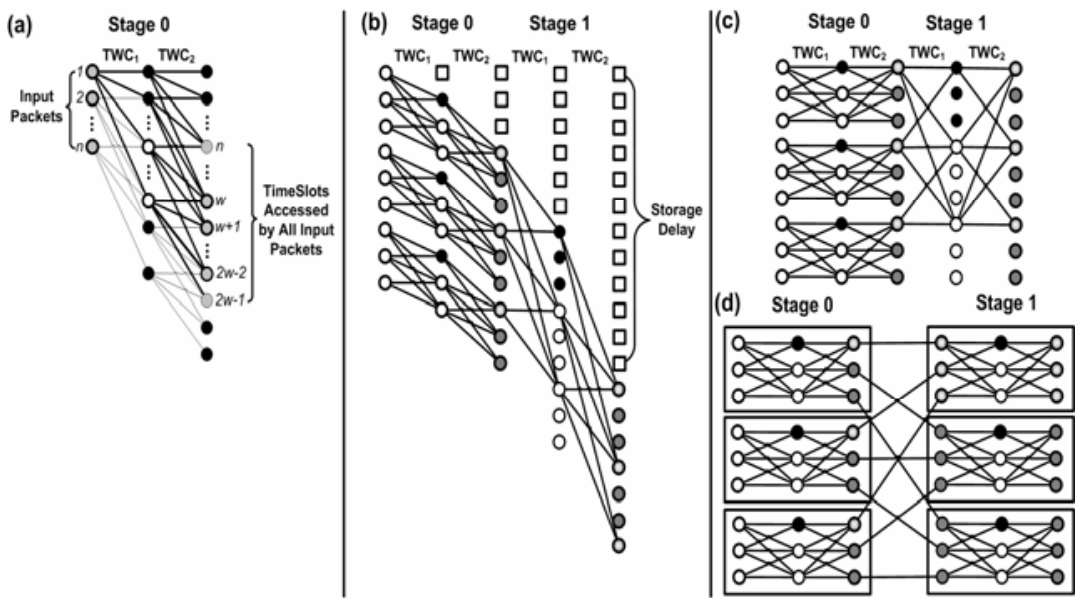

Fig. 2. (a) Formation of the $n x n$ switch on the TTG. (b) Formation of the $\log _{\mathrm{n}}$-Benes network on the TTG. (c) Representation without delays. (d) Equivalent representation. It is assumed that there are four available wavelengths in (b)-(d).

The building blocks of the $\log _{\mathrm{n}}$-Benes graph are $n \mathrm{x} n$ switches, and thus the first step for constructing it, is to determine the switch size. In a previous communication, we had proposed the deployment of a single TWC per stage [4] for constructing the switches. This resulted in poor wavelength utilization, which amounted to approximately $50 \%$ of the available wavelengths, for the switch formation. Thus, the number of available wavelengths limited by the relation of the single-dot bandwidth and the detuning between adjacent wavelengths is of key importance. Therefore, it is proposed to double the number of TWCs that are required per stage, with objective to achieve almost $100 \%$ wavelength utilization when forming the $n \times n$ switches.

The switches are formed out of time transitions on the TTG, as shown in Fig. 2(a), which corresponds to the first stage (stage 0) of the buffer. A packet that has arrived at the input of stage 0 during the first timeslot may only access timeslots $\{1, \ldots, w\}$ at the output of the first TWC, since time transitions to previous timeslots are not allowed. In a similar fashion, the timeslots that are accessible by the aforementioned packet at the output of the second TWC are limited to $\{1, \ldots, 2 w-1)\}$. If $n$ successive input packets are considered, then the timeslots at the output of the first and second TWC that are accessible by all input packets are $\{n, \ldots, w\}$ and $\{n, \ldots, 2 w-1\}$, respectively.

The switch formation requires that the output timeslots that are accessible by all input packets exceed the number of packets themselves, so that the nxn switches may be always formed on the TTG. This is equivalent to 
$2 \cdot w-1-n+1 \geq n \Rightarrow n_{\max }=w$.

Moreover, the interconnection network that corresponds to the $n x n$ switches must be nonblocking, so that packets do not arrive simultaneously at a TWC and are therefore not lost. This is satisfied by ensuring that there are at least two disjoint paths between all input and output timeslot nodes inside the switch. To ensure this, we take into account that the midnodes (nodes at the output of the first TWC) that are accessible to all input nodes are $\{n, \ldots, w\}$. Provided that there are at least two fully accessible mid-nodes, shown in white colour in Fig. 2(a), there are always at least two disjoint interconnection paths towards the mid-nodes of the switch, and as a result

$$
w-n+1 \geq 2 \Rightarrow n_{\max }=w-1 .
$$

Additionally, the existence of the two disjoint paths between the mid-nodes and the output nodes is assured when the output nodes are limited to $\{w, \ldots, 2(w-1)\}$. The switch is therefore formed (a) after selecting $n-2$ mid-nodes that are symmetrically located above and below the two fully accessible mid-nodes when $n$ is even, or (1) after selecting $\frac{n-1}{2}$ and $\frac{n-3}{2}$ mid-nodes above and bellow the fully accessible mid-nodes, respectively, when $n$ is odd. Eq. (3) shows that almost full utilization of the available wavelengths has been achieved with the proposed buffer architecture.

The next step for constructing the $\log _{n}$-Benes graph is to determine the time transitions that form the graph's switches in the respective stages. The process is shown in Fig. 2(b)(d) for the first and second stage of the buffer. The formation of the $\log _{n}$-Benes graph crossbars requires that at each stage $i$, time-transitions connect timeslots that are located $n^{i}$ positions apart. This corresponds to setting the time delays, in timeslots, equal to

$$
D(i, j)=j \cdot n^{i}, \quad i=0, \ldots, m-1, \quad j=0, \ldots, w-1 .
$$

The delays account for all time transitions on the TTG, even though not all transitions contribute to the formation of the virtual switches. The inactive transitions introduce a constant delay after which the output timeframe commences (white squares in Fig. 2(b)). At the output of each buffer stage, the delay equals

$$
\Delta_{i}=n^{i+1}, \quad i=0, \ldots, m-1
$$

timeslots and as a result the delay that the packets experience when traversing the buffer is

$$
\Delta=\sum_{i=0}^{m-1} n^{i+1}+\sum_{i=0}^{m-2} n^{i+1}=n \cdot \frac{n^{m}+n^{m-1}-2}{n-1} .
$$

Eq. (5) may be viewed as a constant buffer access time. 


\subsection{Buffer Control}

Following the discussion of subsection 2.1, packets are buffered after being converted to the appropriate internal wavelengths and accessing the respective delay lines at each programmable delay stage. As a result, buffering requires that the state of wavelength converters has to be set prior to sending the packets to the buffer. From a TTG perspective setting the internal wavelengths is equivalent to calculating the state of the switches in all intermediate stages of the $\log _{n}$-Benes graph of in Fig. 2 (d) so that the input packet sequence is routed to the respective output sequence.

To perform routing in a $\log _{n}$-Benes graph, we have proposed a modified parallel routing algorithm [4] that extends the parallel routing algorithm on a binary Benes graph [3]. The algorithm involved setting the state of the outermost switches (at stages 0 and s-1) of the Benes graph given the respective packet sequences. The outermost switches are then omitted, and the remaining network is partitioned into multiple Benes graphs of reduced size. The algorithm is recursively applied on the resulting graphs until the state of all switches is set.

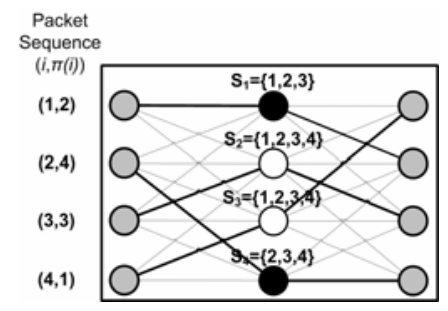

Fig. 3. Routing inside the $n \times n$ switches. It is assumed that there are five available wavelengths

Having determined the state of the switches, it remains to calculate the interconnection pattern inside the switches. We consider that each mid-node $k$ of the switch is described by a set $S_{k}$ that contains the input and output nodes it is connected to. Due to the symmetry of the switch, mid-nodes always connect to the same group of input and output nodes. Supposing that input node $i$ must connect to output node $\pi(i)$, there are at least two midnodes that allow for this connection, since there are at least two disjoint paths between input and output nodes. The mid-nodes $k$ that enable this connection satisfy

$$
(i, \pi(i)) \in S_{k}
$$

An algorithm for selecting the mid-nodes so that all input-to-output connections are performed over disjoint paths (without collisions) is illustrated in Fig. 3. The algorithm involves the following steps:

(a) For a given node pair (i, $\pi(i))$ find all available sets $S_{k}$ that satisfy Eq. (7).

(b) Select the set $S_{k}$ with the smallest number of elements for the node pair.

(c) Proceed to the next node pair $(i+1, \pi(i+1))$ and repeat.

The proposed algorithm calculates hops $(i, k)$ and $(k, \pi(i))$ for all connections $(i, \pi(i))$ inside the switch. The calculated hops correspond to the wavelengths that are provided by the two TWCs of each respective stage. Following the discussion of subsection 2.1 on the switch formation, hop $(i, k)$ corresponds to wavelength 


$$
\begin{aligned}
& \lambda_{\frac{n+1}{2}+k-i}, n \text { is odd } \\
& \frac{\lambda_{\frac{n+2}{2}+k-i},}{n} \text { is even. }
\end{aligned}
$$

In a similar fashion, hop $(i, \pi(i))$ corresponds to wavelength

$$
\begin{aligned}
& \lambda_{\frac{n+3}{2}+\pi(i)-k}, n \text { is odd } \\
& \lambda_{\frac{n+2}{2}+\pi(i)-k}, n \text { is even. }
\end{aligned}
$$

\section{Simulation of the Buffer Architecture}

\subsection{Description of the QDSOA based TWC subsystem}

The proposed buffer architecture aims operation at $160 \mathrm{~Gb} / \mathrm{s}$ line-rate, therefore the TWC realization is based on QDSOA technology which can support high bit rates without patterning effects as opposed to bulk and quantum well SOAs. Fig.4a illustrates the TWC configuration which is based on the cross-gain modulation (XGM) effect of the saturated gain of each amplifier. Two cascaded QDSOA devices have been used to enable wavelength conversion between the modulating pump and continuous wave (cw) probe signals, respectively. Both pump and probe signals are assumed to co-propagate the device. The available wavelengths must fit within the single-dot bandwidth for efficient XGM to occur. Typical values of the homogeneous broadening of QDSOAs at room temperature are $10 \mathrm{meV}-20 \mathrm{meV}$ [5]. In the present study this value corresponds to $16 \mathrm{meV}$, that is $31 \mathrm{~nm}$ at the $1.55 \mathrm{um}$ window. At $160 \mathrm{~Gb} / \mathrm{s}$ the detuning frequency between adjacent wavelengths has been assumed $640 \mathrm{GHz}$ to prevent spectral overlap and it is equivalent to $5.1 \mathrm{~nm}$ for operation at $1.55 \mathrm{um}$. A set of four available wavelengths distributed evenly with respect to the center of the homogeneous broadening has been chosen. The latter coincides with the center of the inhomogeneously broadened gain profile of the QDSOAs located at $\lambda_{\mathrm{c}}=$ $1.55 \mathrm{um}$. It should be noted that, $\lambda_{\mathrm{c}}$ is used as a dummy wavelength for intermediate conversion between the two devices. The available wavelengths are related to $\lambda_{\mathrm{c}}$ as follows: $\left\{\lambda_{1}=\lambda_{\mathrm{c}}-2 \Delta \lambda, \lambda_{2}=\lambda_{\mathrm{c}}-\Delta \lambda, \lambda_{3}=\lambda_{\mathrm{c}}+\Delta \lambda, \lambda_{4}=\lambda_{\mathrm{c}}+2 \Delta \lambda\right\}$, where $\Delta \lambda=5.1 \mathrm{~nm}$ corresponds to the detuning between adjacent wavelengths including $\lambda_{\mathrm{c}}$.

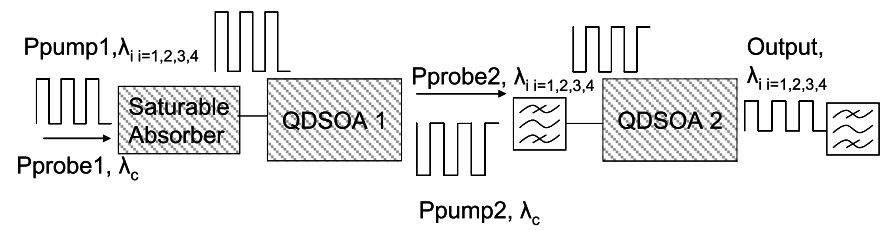

(a)

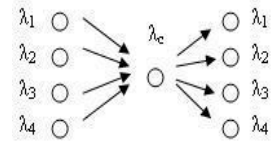

(b)

Fig. 4. (a) Configuration setup of TWC consisting of two QDSOAs in cascade. (b) Available wavelengths within homogeneous broadening of single-dot gain. 
The input signal represented by $\mathrm{P}_{\text {pump1 }}$ carries the binary data at one of the available wavelengths and is used as a pump signal to modulate the carrier density and subsequently the gain of the first QDSOA (QDSOA1). A (cw) probe signal represented by $\mathrm{P}_{\text {probel }}$ and located at $\lambda_{c}$, enters the same device experiencing the gain modulation. Effectively, the binary information carried by $\mathrm{P}_{\text {pump } 1}$ is copied to the (cw) signal with inversed polarity. The output of QDSOA1 constitutes the pump signal that modulates the gain of QDSOA2 and it represented by $\mathrm{P}_{\text {pump2 }}$. A second (cw) signal enters the second QDSOA to achieve wavelength conversion from $\lambda_{c}$ to one of the available wavelengths. The output of the second QDSOA constitutes the output of the TWC. It is noteworthy that, tunable filters are placed at the output of each QDSOA in order to cut off the unnecessary input pump signals. For the specific simulations the device has been assumed as a simple passive element of $2 \mathrm{~dB}$ loss. Additional losses of $6 \mathrm{~dB}$ have been included for the MUX/DEMUX elements giving rise to $8 \mathrm{~dB}$ losses in total.

A saturable absorber is used at the input of each TWC to scale down the extinction ratio degradation along the cascaded subsystems in the buffer architecture. The specific element is also based on QD technology and therefore exhibits very fast response time $(\sim 1.5 \mathrm{psec})[6]$ which enables support of signal processing applications at line-rates of $160 \mathrm{~Gb} / \mathrm{s}$. A simple static transfer function is needed to represent the reshaping characteristic of the device [7]. The latter is introduced through the loss parameter $\alpha(t, P)$ that tracks the signal envelope according to $\alpha(t, P)=\alpha_{0} /\left(1+P(t) / P_{\text {sat }}\right)$, where $P_{\text {sat }}$ is the saturation power $\alpha_{0}$ and is the steady state loss being $-0.1 \mathrm{~dB}$ in this case. Extensive simulations have indicated an optimum value of $+20 \mathrm{dBm}$ for the $P_{\text {sat }}$ parameter.

The rate equation model presented in [8] has been employed, to evaluate the performance of the QDSOA devices in this configuration setup. This is a generalization of the approach used to model bulk/QW devices based on four main assumptions. Firstly, the quantum dots are spatially isolated exchanging carriers only through the wetting layer. Secondly, the dots were grouped together by their resonant frequency in order to treat the spectral hole in the inhomogeneously broadened gain spectrum. Thirdly, the wavelength separation between the pump and probe signals depends on the width of homogeneous broadening of the single-dot gain. Finally, carrier dynamics within the dots is described by four rate equations corresponding to the energy levels of the wetting layer, the continuum state, the excited state and the ground state.

\subsection{Results and discussion}

This section presents the results of the physical layer dimensioning of the proposed buffer architecture. In depth optimization analysis has been carried out to determine the maximum number of stages that can be cascaded. To evaluate the signal quality along the cascade two different metrics as figure of merit functions have been used, corresponding to the Q-factor and the extinction ratio. The Q-factor ratio is directly related to the actual Bit-Error-Rate of the system, only when the signal degradation follows Gaussian statistics. Although this does not happen in the present case, this 
metric can still be used to reflect the efficiency of that process, due to the regenerative capabilities of the TWCs.

The operating conditions for the TWC subsystem have been decided after extensive simulations where various parameters have been optimized such as the active length and the current density of each QDSOA [9]. The main objective is to achieve ultrafast XGM operation and to prevent bit patterning phenomena. For the purpose of the present work, the simulations have been performed assuming input current density $36 \mathrm{kA} / \mathrm{cm}^{2}$ to ensure high carrier population at the upper energy states of the quantum-dots, which act as a reservoir of carriers for the lower energy states. Also, the QDSOAs have been assumed $10 \mathrm{~mm}$ long and the average power of the pump channel has been $+27 \mathrm{dBm}$. The corresponding pulse stream consists of 2-psec $1^{\text {st }}$ order Gaussian pulses, modulated by a $2^{7}-1$ PRBS bit pattern at $160 \mathrm{~Gb} / \mathrm{s}$. Also, a limited amount of amplitude distortion corresponding to $\mathrm{Q}_{\text {in }}=10$, has been assumed at the input, whilst the extinction ratio is $13 \mathrm{~dB}$. A detailed set of the QDSOA simulation parameters used in this study, can be found in [8].

Important design issue for the subsystem of the TWC is the identification of the optimum power levels for the probe wavelengths at the input of the first and the second QDSOA represented by $\mathrm{P}_{\text {probe1 }}$ and $\mathrm{P}_{\text {probe2 }}$, respectively. The goal of such an optimization process has three aspects. The first is to maintain the extinction ratio at the output at the same level as that of the input. The use of the saturable absorber has a significant impact on this. Secondly, the operation of the TWC should be regenerative, which practically indicates improvement of the Q-factor ratio. Finally, the output signal should maintain a high power level to ensure an efficient XGM performance at the following TWC.

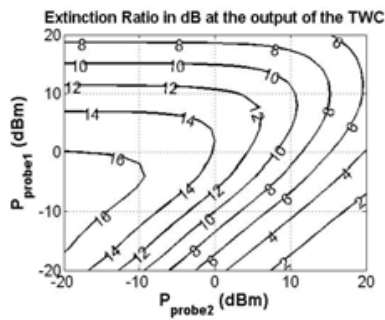

(a)

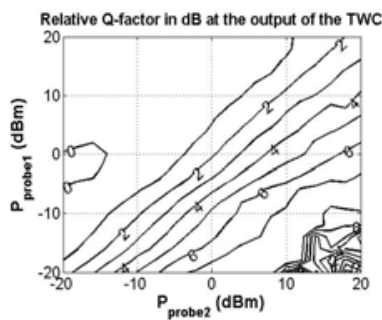

(b)

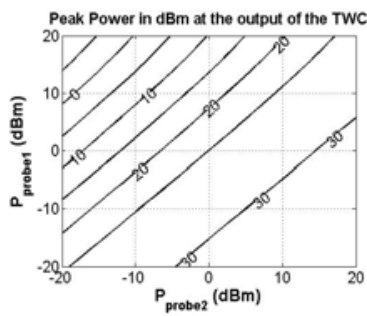

(c)

Fig. 5. (a) Extinction ratio (b) Relative Q-factor and (c) Peak power, at the output of the TWC

Fig. 5 (a-c) illustrates the extinction ratio, the relative Q-factor improvement and the peak power of the output pulse-stream as a function of $\mathrm{P}_{\text {probel }}$ and $\mathrm{P}_{\text {probe2 }}$, respectively. It is clear that, extinction ratio is severely degraded as $\mathrm{P}_{\text {probe2 }}$ increases, which has also been demonstrated for SOAs in XGM operation [10]. On the other hand, high power level of $\mathrm{P}_{\text {probel }}$ is required to achieve sufficient pump power at the input of the second QDSOA and satisfactory extinction ratio at the output of the TWC. The regenerative efficiency of the subsystem is related to the Q-factor which shows opposite behaviour to the extinction ratio. In particular, increased level of $\mathrm{P}_{\text {probe2 }}$ will accelerate the recovery of the saturated gain of QDSOA2, which in turn results in reduction of patterning effects of the output pulse stream. It has already been mentioned that the output power level of the TWC is very important because it 
feeds the next TWC. Fig. 5c illustrates that, the peak power of $+30 \mathrm{dBm}$ is maintained around a region where the extinction ratio has decreased to around $8 \mathrm{~dB}$ whereas, the $\mathrm{Q}$-factor has increased about $6 \mathrm{~dB}$ with respect to the input value $\left(\mathrm{Q}_{\text {in }} \approx 10\right)$.

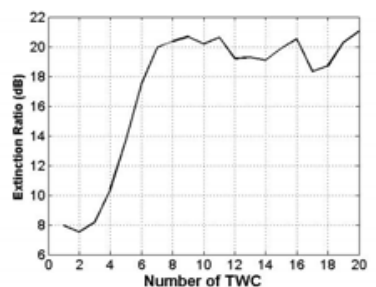

(a)

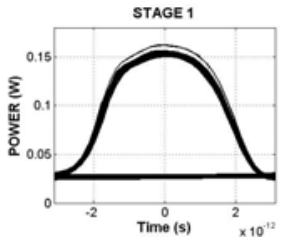

(c)

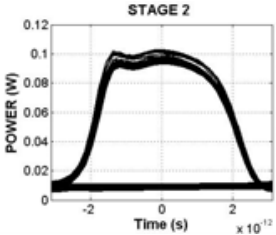

(d)

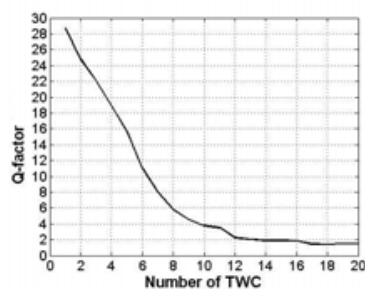

(b)

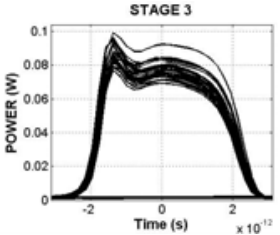

(e)

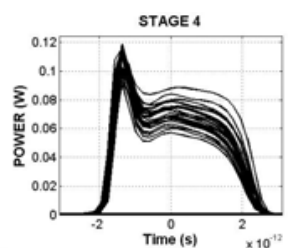

(f)

Fig. 6. (a-b) Extinction ratio and Q-factor as a function of the number of cascaded TWCs (c-f) eye diagrams at the output of the $1^{\text {st }}, 2^{\text {nd }}, 3^{\text {rd }}$ and $4^{\text {rth }}$ stage.

It is noteworthy that, the operating conditions change when the TWCs operate in cascade. The worst case scenario has been considered here, in which conversion from $\lambda_{1}$ to $\lambda_{4}$ and $\lambda_{4}$ to $\lambda_{1}$ occurs successively at each stage. Fig. 6 illustrates the extinction ratio and the Q-factor as a function of the number of cascaded TWCs as well as the eye diagrams after the first four stages of the buffer architecture. The operating conditions have been identified $\{-15 \mathrm{dBm}, 0 \mathrm{dBm}\}$ for $\mathrm{P}_{\text {probe } 1}$ and $\mathrm{P}_{\text {probe } 2}$, respectively. The performance of the subsystem in terms of extinction ratio has improved along the cascade owing to the use of the saturable absorber which suppresses the amplitude variation at the spaces. Furthermore, the extinction ratio increases towards a steady level of $20 \mathrm{~dB}, 7 \mathrm{~dB}$ above the extinction ratio at the input of the first converter $(13 \mathrm{~dB})$.

However, the performance of the cascade is limited by the degradation of the Qfactor at the output of each converter. The eye diagrams of the output pulse streams illustrate that the pulse width increases along the cascade introducing a duty cycle distortion. Moreover, overshooting is experienced by the leading edge of the pulse which is related to the fact that the leading edge saturates the amplifier so that the trailing edge experiences less gain than the leading edge. This effect becomes more severe along the cascade and limits the performance of the buffer to 3 stages. In addition, the output peak power along the cascade is reduced and the Q-factor decreases. In particular, at the output of the third stage, that is, at the output of the sixth TWC, the Q-factor is equal to its input value indicating error-free operation. It is clear that, the information at the output of the fourth stage is distorted. The number of packets that the proposed buffer architecture can support by use of four available wavelengths and $\mathrm{s}=3$ stages is 9 , as derived from equation (1). 


\section{Conclusion}

In this communication we have demonstrated the applicability of tunable wavelength converters (TWC) based on QDSOA technology to the implementation of an ultra-high speed optical packet switching buffer. The buffer architecture consists of cascaded stages of two TWC in series and makes use of all available wavelengths located within the homogeneous broadening of the single-dot gain of the QDSOA device. Effectively, the number of cascaded TWC needed to serve a certain number of input packets is significantly decreased, reducing the cost in terms of hardware implementation of the buffer architecture.

The cascadability results of the TWC configuration have been studied in terms of extinction ratio and Q-factor to ensure good quality of the output signal. The extinction ratio at the output of the TWC has been significantly improved by use of the saturable absorber. Finally, error-free operation has been shown up to 3 stages of the buffer architecture and thus, support of 9 input packets.

Acknowledgments. The work was supported by the Operational Program for Educational and Vocational Training (EPEAEK), PYTHAGORAS II Program and by EU via the IST/NoE e-Photon/ONe+ project, COST 291 and the project TRIUMPH (IST-027638 STP).

\section{References}

1. Borri, P., Langbein, W., Hvam, J.M., Heinrichdorff, F., Mao, M.-H., Bimberg, D.: Ultrafast gain dynamics in InAs-InGaAs quantum-dot amplifiers. IEEE Photon. Technol. Lett. 12 (2000) 594596

2. Hunter, D.K., Smith, D.G.: New architectures for optical TDM switching. IEEE/OSA J. Lightwave Technol. 11 (1993) 495-511

3. Lee, T.T., Liew, S.Y.: Parallel Routing Algorithms in Benes-Clos Networks. IEEE Trans. Commun. 50 (2002) 1841-47

4. Yiannopoulos, K., Varvarigos, E., Vlachos, K.: Multiple-Input Buffer and Shared Buffer Architectures for Optical Packet and Burst Switching Networks. IEEE/OSA J. Lightwave Technol. submitted

5. Sugawara, M., et al.: Effect of homogeneous broadening of optical gain on lasing spectra in selfassembled InxGa1-xAs/GaAs quantum dor lasers. Physical Review B 61 (2000) 7595-7603

6. Massoubre, D.: High Speed Switching Contrast Quantum Well Saturable Absorber for $160 \mathrm{~Gb} / \mathrm{s}$ Operation. Conference on Lasers \& Electro-Optics (CLEO), 2005, CThD3

7. Audouin, O., Pallise, E., Desurvire, E., Maunand E.: Use of Fast In-Line Saturable Absorbers in Wavelength-Division-Multiplexed Solitons Systems. IEEE Photon. Technol. Lett. 10 (1998) 828-830

8. Sugawara, M. Akiyama, T., et al.: Quantum-dot semiconductor optical amplifiers for high-bitrate signal processing up to $160 \mathrm{~Gb} / \mathrm{s}$ and a new scheme of 3R regenerators. Meas. Sci. Technol. 13 (2002) 1683-1691

9. Spyropoulou, M., et al.: Study of Multi-wavelength Regeneration based on Quantum Dot Semiconductor Optical Amplifiers. IEEE Photon. Technol. Lett. submitted

10. Lee, H., et al.: Theoretical Study of Frequency Chirping and Extinction Ratio of WavelengthConverted Optical Signals by XGM and XPM using SOA's. IEEE J. of Quantum Electronics 35 (1999) 1213-1219 